\title{
CHIRAL THIRRING-WESS MODEL WITH FADDEEVIAN REGULARIZATION
}

\author{
Anisur Rahamar* \\ Hooghly Mohsin College, Chinsurah, Hooghly - 712101, West Bengal, India
}

\begin{abstract}
Replacing vector type of interaction of the Thirring-Wess model by the chiral type a new model is presented which is termed here as chiral Thirring-Wess model. Ambiguity parameters of regularization is so chosen that the model falls into the Faddeevian class. The resulting Faddeevian class of model in general do not possess Lorentz invariance. However we can exploit the arbitrariness admissible in the ambiguity parameters to relate the quantum mechanically generated ambiguity parameters with the classical parameter involved in the masslike term of the gauge field which helps to maintain physical Lorentz invariance instead of the absence of manifestly lorentz covariance of the model. The the phase space structure and the theoretical spectrum of this class of model has been determined through Dirac's method of quantization of constraint system.
\end{abstract}

PACS numbers: 11.10.Ef, 11.30.Rd

\section{INTRODUCTION}

Generation of mass without violating the gauge invariance is a celebrated physical principle. In this context Schwinger model acquired a significant position in lower dimensional field theory [1 7]. Here photon acquires mass via a kind of dynamical symmetry breaking keeping the gauge symmetry of the model intact. Few years later, Thirring and Wess proposed a two dimensional field theoretical model where also photon acquires mass but the gauge symmetry of the model breaks down at the classical level [8]. Recently, an attempt has been made in [9], for systematic functional integral bosonization of this mode. After few years of presentation of the Thirring-Wess model, chiral generation of Schwinger model was proposed in [10]. However, the model remaimd less attractive over a long period because of its non-unitary problem. But it attracted attentions and gradually acquired a significant position in lower dimensional field theory after the work of Jackiw and Rajaraman where they became able to remove the non-unitary problem taking into account the electromagnetic anomaly into that model [11]. The welcome entry of the anomaly and a suitable exploitation of the ambiguity involved therein made Jackiw-Rajaraman version of Chiral Schwinger model 11-16] along with the other independent regularized version of that model [17 20] interesting as well as attractive in lower dimensional field theory regime. Not only in the chiral Schwinger model but also vector Schwinger model [1] turns into the so called non-confining Schwinger model when anomaly enters into it 21]. A suitable exploitation of the ambiguity involved here has been made to restore the lost gauge invariance of this model in [22]. This present work will also be a display of exploitation of ambiguity parameter in the so called chiral Thirring-Wess model with Faddeevian anomaly in order to get a lorentz invariant theory where Lorentz invariance was absent to start with.

In the Thirring-Wess model the authors considered a theory of massles fermion interacting with massive vector field in two dimension. It can be thought of as a study of QED, viz., Schwinger model [1, 2] replacing Maxwell's field by Proca and that very replacement breaks the gauge symmetry at the classical level but a consistent field theoretical model gets birth. It is true that the so called non-confining Schwinger model [21, 23] is a structurally equivalent gauge non-invariant model to the Thirring-Wess mode but there lies a crucial difference between these two. In the Thirring-Wess mode the masslike term for the gauge field was included at the classical level however in the the so called non-confining Schwinger model the same type of masslike term gets involved through one loop correction which contains an ambiguity parameter too. In [22], we have noticed a competition between the classically included masslike term and quantum mechanically generated masslike term in connection with gauge symmetry restoration of the so called non confining Schwinger model.

An attempt has been made to get chiral generation of the Thirring-Wess mode in the similar way the chiral generation of the Schwinger model was made in [10]. How anomaly becomes useful in the present context to get a consistent and physically sensible theory that we would like to address here for the Faddeevian class of regularization. So we replace vector interaction of the Thirring-Wess model by the chiral one that generates a new model which we would like to term as chiral Thirring-Wess model. The resulting model does not possess Physical lorentz invariance for all admissible regularization. Regularization is needed in order to remove the divergence of the fermionic determinant that appears in the process of bosonization integrating out the fermions one by one. It would be of interest whether

*Electronic address: 1. anisur.rahman@saha.ac.in, 2. manisurn@gmail.com 
the absence of physical lorentz invariance in the so called Chiral Thirring-Wess model with Faddeevian class of regularization gets restored in a manner gauge invariance was restored in [22] exploiting the arbitrariness in the ambiguity parameter. How does anomaly in general and regularization ambiguity in particular come in use in this type of investigation that is the main objective of the present work?

\section{CHIRAL GENERATION OF THE THIRRING-WESS MODEL WITH FADDEEVIAN REGULARIZATION}

The so called Chiral Thirring Wess model can be framed by the following generating functional

$$
Z[A]=\int d \psi d \bar{\psi} e^{\int d^{2} x \mathcal{L}_{f}}
$$

with

$$
\begin{aligned}
\mathcal{L}_{f} & =\bar{\psi} \gamma^{\mu}\left[i \partial_{\mu}+e \sqrt{\pi} A_{\mu}\left(1-\gamma_{5}\right)\right] \psi \\
& =\bar{\psi}_{R} \gamma^{\mu} i \partial_{\mu} \psi_{R}+\bar{\psi}_{L} \gamma^{\mu}\left(i \partial_{\mu}+2 e \sqrt{\pi} A_{\mu}\right) \psi_{L}
\end{aligned}
$$

Here dynamics of the $A_{\mu}$ field is governed by he Proca field and the lagrangian of which is given by

$$
\mathcal{L}_{\text {Praca }}=\frac{1}{4} F_{\mu \nu} F^{\mu \nu}+\frac{1}{2} m^{2} A_{\mu} A^{\mu}
$$

Note that, we have replace the vector type of interaction $\bar{\psi} \gamma_{\mu} \psi A^{\mu}$ by the chiral type $\bar{\psi} \gamma_{\mu}\left(1+\gamma_{5}\right) \psi A^{\mu}$. Let us now proceed with the fermionic part of the lagrangian density. The right handed fermion remains uncoupled in this type of chiral interaction. So integration over this right handed part leads to field independent counter part which can be absorbed within the normalization. Integration over left handed fermion leads to

$$
\begin{aligned}
Z[A] & =\int d \psi_{L} d \bar{\psi}_{L} \bar{\psi}_{L} \gamma^{\mu}\left(i \partial_{\mu}+2 e \sqrt{\pi} A_{\mu}\right) \psi_{L} \\
& =\exp \frac{i e^{2}}{2} \int d^{2} x A_{\mu}\left[M_{\mu \nu}-\left(\partial^{\mu}+\tilde{\partial}^{\mu}\right) \frac{1}{\square}\left(\partial^{\nu}+\tilde{\partial}^{\nu}\right)\right] A_{\nu}
\end{aligned}
$$

Where $M_{\mu \nu}=a g_{\mu \nu}$, for Jackiw-Rajaraman type of regularization and the model remains manifestly lorentz covariant for this setting. The parameter $a$ represents the regularization ambiguity here. In general, the elements of $M_{\mu \nu}$ can take any arbitrary values. However, the model looses both its solvability and Lorentz invariance in that situation. We consider here a symmetric form of $M_{\mu \nu}$ :

$$
M_{\mu \nu}=\left(\begin{array}{cc}
\tilde{a} & \alpha \\
\alpha & \gamma
\end{array}\right) \delta(x-y) .
$$

Here regularization ambiguity is involved within the parameters $\tilde{a}, \alpha$ and $\gamma$. These parameters gets involved in order to remove the divergence in the fermionic determinant since the evaluation of the determinant needs a one loop correction [17, 19, 20]. It may be the situation that all the parameters are not independent for the model to be physically sensible.

This generating functional (4) when written in terms of the auxiliary field $\phi(x)$ it turns out to the following

$$
Z[A]=\int d \phi e^{i \int d^{2} x \mathcal{L}_{B},}
$$

with

$$
\begin{aligned}
\mathcal{L}_{B} & =\frac{1}{2}\left(\partial_{\mu} \phi\right)\left(\partial^{\mu} \phi\right)+e\left(g^{\mu \nu}-\epsilon^{\mu \nu}\right) \partial_{\nu} \phi A_{\mu}+\frac{1}{2} e^{2} A_{\mu} M^{\mu \nu} A_{\nu} \\
& =\frac{1}{2}\left(\dot{\phi}^{2}-\phi^{\prime 2}\right)+e\left(\dot{\phi}+\phi^{\prime}\right)\left(A_{0}-A_{1}\right)+\frac{1}{2} e^{2}\left(\tilde{a} A_{0}^{2}+2 \alpha A_{0} A_{1}+\gamma A_{1}^{2}\right) .
\end{aligned}
$$

So the total lagrangian density of our present interest is

$$
\begin{aligned}
\mathcal{L} & =\mathcal{L}_{B}+\mathcal{L}_{\text {Praca }} \\
& =\frac{1}{2}\left(\partial_{\mu} \phi\right)\left(\partial^{\mu} \phi\right)+e\left(g^{\mu \nu}-\epsilon^{\mu \nu)} \partial_{\nu} \phi A_{\mu}+\frac{1}{2} e^{2} A_{\mu} M^{\mu \nu} A_{\nu}-\frac{1}{4} F_{\mu \nu} F^{\mu \nu}+\frac{1}{2} m^{2} A_{\mu} A^{\mu}\right. \\
& =\frac{1}{2}\left(\dot{\phi}^{2}-\phi^{\prime 2}\right)+e\left(\dot{\phi}+\phi^{\prime}\right)\left(A_{0}-A_{1}\right)+\frac{1}{2} e^{2}\left(\tilde{a} A_{0}^{2}+2 \alpha A_{0} A_{1}+\gamma A_{1}^{2}\right) \\
& +\frac{1}{2}\left(\dot{A}_{1}^{2}-A_{0}^{\prime 2}\right)+\frac{1}{2} m^{2}\left(A_{0}^{2}-A_{1}^{2}\right)
\end{aligned}
$$


Here the masslike terms for gauge fields in the lagrangian density (8) is

$$
\begin{aligned}
\mathcal{L}_{\text {mass }} & =\frac{1}{2} e^{2}\left(\tilde{a} A_{0}^{2}+2 \alpha A_{0} A_{1}+\gamma A_{1}^{2}\right)+\frac{1}{2} m^{2}\left(A_{0}^{2}-A_{1}^{2}\right) \\
& =\frac{1}{2} e^{2}\left[\left(\tilde{a}+\frac{m^{2}}{e^{2}}\right) A_{0}^{2}+2 \alpha A_{0} A_{1}+\left(\gamma-\frac{m^{2}}{e^{2}}\right) A_{1}^{2}\right] .
\end{aligned}
$$

This Lagrangian in general fails to provide Poincaré invariant equations of motion. Ambiguity in the regularization allows us to put any condition unless it violates any physical principle of the theory. We thus set $\tilde{a}+\frac{m^{2}}{e^{2}}=1$ in order to make the lagrangian free from the quadratic term of $A_{0}$. With this choice the constraints of the theory falls under the Faddeevian class [24 27]. It has a deeper meaning and interesting consequences 24 27]. Some other choices may lead to physically sensible theory. It would certainly be the issue of further investigations. Here we would like to keep ourselves confined in the choice that leads to Faddeevian class of constraint structure, to be more precise, Faddeevian class of Gauss law.

\section{CONSTRAINT ANALYSIS AND DETERMINATION OF THE THEORETICAL SPECTRUM}

Let us now proceed with the constraint analysis of the theory. To this end we require to calculate the canonical momenta of the fields involved in the theory. The momentum corresponding to the field $\phi, A_{0}$ and $A_{1}$ respectively are

$$
\begin{gathered}
\pi_{\phi}=\dot{\phi}+e\left(A_{0}-A_{1}\right), \\
\pi_{0}=0, \\
\pi_{1}=\dot{A}_{1}-A_{0}^{\prime} .
\end{gathered}
$$

The hamiltonian obtained through the Legendry transformation is

$$
H_{B}=\int d x\left[\pi_{\phi} \dot{\phi}+\pi_{1} \dot{A}_{1}+\pi_{0} \dot{A}_{0}-\mathcal{L}\right]
$$

which gives the following hamiltonian density

$$
\begin{aligned}
\mathcal{H}_{B} & =\frac{1}{2} \pi_{1}^{2}+\pi_{1} A_{0}^{\prime}+\frac{1}{2}\left[\pi_{\phi}-e\left(A_{0}-A_{1}\right)\right]^{2}+\frac{1}{2} \phi^{\prime 2}-e \phi^{\prime}\left(A_{0}-A_{1}\right) \\
& -\frac{1}{2} e^{2}\left(A_{0}^{2}+2 \alpha A_{0} A_{1}+\left(\gamma-\frac{m^{2}}{e^{2}}\right) A_{1}^{2}\right) .
\end{aligned}
$$

Equation (11) is independent of $\dot{A}_{0}$. So it is the primary constraint of the theory. At this stage it is useful to work with the effective hamiltonian

$$
H_{\text {Beff }}=H+\int d x u \pi_{0} .
$$

Lagrangian multiplier $u$ remains undetermined at this stage. It will be fixed later. The preservation of the primary constraint $\pi_{0} \approx 0$, gives Gauss law as the secondary constraint:

$$
G=\pi_{1}^{\prime}+e\left(\pi_{\phi}+\phi^{\prime}\right)+e^{2}(1+\alpha) A_{1} \approx 0 .
$$

This Gauss law constraint also has to be preserve in time in order to have a consistent theory. The preservation condition of the Gauss law is $\dot{G}(x)=[G(x), H(y)]=0$, and that leads to the following new constraint

$$
(1+\alpha) \pi_{1}+2 \alpha A_{0}^{\prime}+\left(\gamma-\frac{m^{2}}{e^{2}}+1\right) A_{1}^{\prime} \approx 0 .
$$

The preservation of the constraint (17) does not give rise to any new constraint. It fixes the velocity $u$. Note that, the Gauss law constraint (16) is Faddeevian in nature [24 27]. Though the term 'Faddeevian'is very standard in $(1+1)$ dimensional field theory for the reader's benefit we should explain a bit about Faddeevian nature of constraint. If the 
Gauss constraint reflects the presence of Schwinger term like $[G(x), G(y)]=A \delta^{\prime}(x-y)$, where $A$ is a constant, then gauge invariance gets lost and that poses a threat on the quantization of the theory. In Ref. [24, 25], Faddeev initially argued that in spite of the presence of this type of abnormality it is possible to quantize the theory. However, the degrees of freedom would be more in number because no gauge fixing condition is needed. So the quantization of the present theory would be interesting in its own right because the Gauss law $\mathrm{G}(\mathrm{x})$ gives the following Poission bracket

$$
[G(x), G(y)]=2 e^{2}(3+\alpha) \delta^{\prime}(x-y)
$$

which fits with Faddeevian nature. In this context, we would like to mention that if we look towards the Poisson brackets (18) which would be appeared for the usual chiral Schwinger model [11] and the vector Schwinger model [1, 21] we will find that the Scgwinger term will be absent there. In fact, it gives a vanishing contribution for those cases.

The constraints are all weak condition at this stage. If we impose these constraints into the hamiltonian treating them as strong condition, the hamiltonian will be reduced to

$$
H_{R}=\int d x\left[\frac{1}{2} \pi_{1}^{2}+\frac{1}{2 e^{2}} \pi_{1}^{\prime 2}-\alpha \pi_{1} A_{1}^{\prime}+e(1+\alpha) A_{1} \phi^{\prime}+\frac{1}{e} \pi_{1}^{\prime} \phi^{\prime}+\phi^{\prime 2}+\frac{1}{2} e^{2}\left[\alpha^{2}-\gamma+\frac{m^{2}}{e^{2}}\right] A_{1}^{2}\right] .
$$

But we have to keep it in mind that the canonical Poission brackets will be inadequate for this reduced Hamiltonian for computation of equations of motion [28. To get correct equations of motion for this constrained system appropriate Dirac brackets 28] have to be employed in place of ordinary Poisson brackets. It is known that Dirac bracket between the two variables $\mathrm{A}(\mathrm{x})$ and $\mathrm{B}(\mathrm{y})$ is defined by

$$
[A(x), B(y)]^{*}=[A(x), B(y)]-\int\left[A(x) \omega_{i}(\eta)\right] C_{i j}^{-1}(\eta, z)\left[\omega_{j}(z), B(y)\right] d \eta d z,
$$

where $C_{i j}^{-1}(x, y)$ is given by

$$
\int C_{i j}^{-1}(x, z)\left[\omega_{i}(z), \omega_{j}(y)\right] d z=1
$$

Here $\omega_{i}$ 's represents the second class constraints of the theory. With the help of equation (20), the Dirac brackets among the fields $A_{1}, \pi_{1}, \phi$, and $\pi_{\phi}$ are calculated:

$$
\begin{gathered}
{\left[A_{1}(x), A_{1}(y)\right]^{*}=\frac{1}{2 \alpha e^{2}} \delta^{\prime}(x-y),} \\
{[\phi(x), \phi(y)]^{*}=-\frac{1}{4 \alpha} \epsilon(x-y)} \\
{\left[A_{1}(x), \phi(y)\right]^{*}=\frac{1}{2 \alpha e} \delta(x-y)} \\
{\left[A_{1}(x), \pi_{1}(y)\right]^{*}=\frac{\alpha-1}{2 \alpha} \delta(x-y)} \\
{[\pi(x), \pi(y)]^{*}=-\frac{e^{2}}{2 \alpha}(1-\alpha)^{2} \epsilon(x-y)} \\
{[\phi(x), \pi(y)]^{*}=-\frac{e}{4 \alpha}(1-\alpha) \epsilon(x-y)}
\end{gathered}
$$

Making use of the equations (22), (23), (24), (25), (26) and (27) equations of motion for the fields appearing in the reduced hamiltonian (19) are obtained as follows.

$$
\dot{A}_{1}=\frac{\alpha-1}{2 \alpha} \pi_{1}-\frac{1}{2 \alpha}\left(1+\gamma-\frac{m^{2}}{e^{2}}\right) A_{1}^{\prime},
$$




$$
\begin{gathered}
\dot{\pi}_{1}=\pi_{1}^{\prime}-\frac{e^{2}}{2 \alpha}\left((1+\alpha)\left(1-\alpha^{2}\right)-(1-\alpha)\left(\alpha^{2}-\gamma+\frac{m^{2}}{e^{2}}\right)\right) A_{1} . \\
\dot{\phi}=-\phi^{\prime}-\frac{1}{e} \pi_{1}^{\prime}+\frac{e}{2 \alpha}\left(\gamma-2 \alpha^{2}+1-\frac{m^{2}}{e^{2}}\right) A_{1} .
\end{gathered}
$$

After a little algebra, we find that the above three equation reduce to the following Lorentz invariant equations

$$
\left(\square-\frac{(\alpha-1)^{2}}{\alpha} e^{2}\right) \pi_{1}=0,
$$

and

$$
\partial_{+} \eta=0
$$

if we set the following relation of the ambiguity parameters $\alpha$ and $\gamma$ with the classical parameter $m^{2}$

$$
m^{2}=e^{2}(1+\gamma-2 \alpha)
$$

The field $\eta$ in (32) is defined as $\eta=\phi+\frac{\alpha}{e(\alpha-1)}\left(\dot{A}_{1}+A_{1}^{\prime}\right)$.

The setting of the above relation (33) becomes possible without violating any physical principle if we are allowed to exploit the arbitrariness admissible in the ambiguity parameters. We are familiar with this practice in different contexts [11 14, 17, 18, 22, 29 31]. The above settings makes the model not only solvable but also renders an interesting lorentz invariant theoretical spectrum, though to start with lorentz covariance was not manifested in the lagrangian. The equations (31) and (32) suggest that the theoretical spectrum contains a massive boson and a massless boson with a definite chirality. The square of the mass of the massive boson is given by $\tilde{m}^{2}=-\frac{(\alpha-1)^{2}}{\alpha}$. The parameter $\alpha$ must be negative for the mass of the boson to be positive. Of course, one can set the matrix $\stackrel{\alpha}{\mu \nu}_{\mu \nu}$ to start with in such a way such that mass term comes out positive. Since the massless boson appeared in the spectrum has a definite chirality, it can be thought of as the a boson of the opposite chirality to this chiral boson has been eaten up during the process. The eaten up chiral boson, which is equivalent to a chiral fermion in $(1+1)$ dimension is, therefore, can be considered as it has became confined. This scenario would be more transparent when we will study this model imposing a chiral constraint in the following section.

\section{IMPOSITION OF CHIRAL CONSTRAIN}

Chiral boson is a basic ingredient of heterotic string theory. So it would be beneficial to express this model in terms of chiral boson. It is also a matter of investigation whether this model remains solvable after imposition of this constraint. In this context, I should mention that if we impose this type of constraint to any arbitrary model that may bring a disaster so far Lorentz invariance and exactly solvability is concerned. We, therefore, impose a chiral constraint in the model described by the lagrangian density (8) to express the model in terms of chiral boson in a manner it was done in [14] and investigation is carried out towards the study of its solvability and maintenance of its Lorentz invariance.

Let us now proceed to impose the following chiral constraint

$$
\omega(x)=\pi_{\phi}(x)-\phi^{\prime}(x)=0 .
$$

It is a second class constraint itself since

$$
[\omega(x), \omega(y)]=-2 \delta^{\prime}(x-y) .
$$

After imposing the constraint $\omega(x)=0$, into the generating functional we find

$$
\begin{aligned}
Z_{C H} & =\int d \phi d \pi_{\phi} \delta\left(\pi_{\phi}-\phi^{\prime}\right) \sqrt{\operatorname{det}[\omega, \omega]} e^{i \int d^{2} x\left(\pi_{\phi} \dot{\phi}-\mathcal{H}_{B}\right)} \\
& =\int d \phi e^{i \int d^{2} x \mathcal{L}_{C H}}
\end{aligned}
$$

with

$$
\mathcal{L}_{C H}=\dot{\phi} \phi^{\prime}-\phi^{\prime 2}+2 e\left(A_{0}-A_{1}\right) \phi^{\prime}+\frac{1}{2} e^{2}\left[\left(\gamma-\frac{m^{2}}{e^{2}}-1\right) A_{1}^{2}+2(\alpha+1) A_{0} A_{1}\right]
$$


It provides a systematic description of the previous lagrangian (8) in terms of chiral boson [14]. Note that the first two term is the kinetic term of the chiral boson [32 35]. In [14], we found the imposition of this type of chirl constraint on the usual chiral Schwinger model with one parameter class of regularization provided by Jackiw and Rajaraman and a description of the usual chiral Schwinger model in terms of chiral boson resulted in. Here we have got an opportunity of using the same prescription once more. In the following section, we will carry out the hamiltonian analysis of the above lagrangian adding the kinetic energy term for the Proca field with the lagrangian density $\mathcal{L}_{C H}$. Needless to mention that the mass term for Praca field is already incorporated within the mass like terms of the $A$ fields. So the starting lagrangian density in this situation is

$$
\mathcal{L}=\mathcal{L}_{C H}-\frac{1}{4} F_{\mu \nu} F^{\mu \nu}
$$

Here $F_{\mu \nu}$ stands for the field strength for the electromagnetic field. Though this model has a structural similarity with the chiral Schwinger model there lies a crucial difference between these two. Unlike the Chiral Schwinger, model this model contains a classical parameter and we have already seen in the previous section that that very parameter lies in the root to make this model exactly solvable with a Lorentz invariant theoretical spectrum getting mixed up suitability with the ambiguity parameter.

\section{DETERMINATION OF THEORETICAL SPECTRUM AFTER THE IMPOSITION OF CHIRAL CONSTRAINT}

For the determination of theoretical spectrum at first we need to calculate momenta corresponding to the field describing the theory. From the standard definition the momenta corresponding to the field $\pi_{\phi}, \pi_{0}$ and $\pi_{1}$ are found out.

$$
\begin{gathered}
\pi_{\phi}=\phi^{\prime}, \\
\pi_{0}=0, \\
\pi_{1}=\dot{A}_{1}-A_{0}^{\prime} .
\end{gathered}
$$

Using the above equations it is straightforward to obtain the canonical hamiltonian through a Legendry transformation which reads

$$
H_{C}=\int d x\left[\frac{1}{2} \pi_{1}^{2}+\pi_{1} A_{0}^{\prime}+\phi^{\prime 2}-2 e\left(A_{0}-A_{1}\right) \phi^{\prime}-\frac{1}{2} e^{2}\left[\left(\gamma-\frac{m^{2}}{e^{2}}-1\right) A_{1}^{2}+2(1+\alpha) A_{0} A_{1}\right)\right] .
$$

Equation (39) and (40) are the primary constraints of the theory. Therefore, the effective hamiltonian is given by

$$
H_{E F F}=H_{C}+\tilde{u} \pi_{0}+v\left(\pi_{\phi}-\phi^{\prime}\right),
$$

where $\tilde{u}$ and $v$ are two arbitrary lagrange multiplier. The constraints obtained in (39) and (40) have to be preserve in order to have a consistent theory. The preservation of the constraint (40), leads to a new constraint which is the Gauss law of the theory:

$$
G=\pi_{1}^{\prime}+2 e \phi^{\prime}+e^{2}(1+\alpha) A_{1} \approx 0 .
$$

The preservation of constraint (39), though does not give rise to any new constraint it fixes the velocity $v$ and that comes out to be

$$
v=\phi^{\prime}-e\left(A_{0}-A_{1}\right) .
$$

The preservation of the Gauss law constraint $\dot{G}=0$, again gives rise to a new constraint

$$
(1+\alpha) \pi_{1}+2 \alpha A_{0}^{\prime}+\left(\gamma-\frac{m^{2}}{e^{2}}+1\right) A_{1}^{\prime} \approx 0 .
$$

No new constraints comes out from the preservation of (46). So the phase space of the theory contains the following four constraints.

$$
\omega_{1}=\pi_{0} \approx 0
$$




$$
\begin{gathered}
\omega_{2}=\pi_{1}^{\prime}+e \phi^{\prime}+e^{2}(1+\alpha) A_{1} \approx 0, \\
\omega_{3}=(1+\alpha) \pi_{1}+2 \alpha A_{0}^{\prime}+\left(\gamma-\frac{m^{2}}{e^{2}}+1\right) A_{1}^{\prime} \approx 0, \\
\omega_{4}=\pi_{\phi}-\phi^{\prime} \approx 0 .
\end{gathered}
$$

The four constraints (47), (48), (49) and (50) are all weak condition up to this stage. Treating this constraints as strong condition we obtain the following reduced hamiltonian.

$$
H_{R}=\int d x\left[\frac{1}{2} \pi_{1}^{2}+\frac{1}{4 e^{2}} \pi_{1}^{\prime 2}+\frac{1}{2}(\alpha-1) \pi_{1}^{\prime} A_{1}+\frac{1}{4} e^{2}\left[(1-\alpha)^{2}-2\left(1+\gamma-\frac{m^{2}}{e^{2}}\right)\right] A_{1}^{2}\right] .
$$

As has been stated earlier we need to calculate Dirac bracket in order to proceed for further analysis because this reduced Hamiltonian will give correct equations of motion only when Dirac brackets will be used for computation. We find that,

$$
\begin{aligned}
C_{i j}(x, y)=\left[\omega_{i}(x), \omega_{j}(y)\right] & = \\
& \left(\begin{array}{cccc}
0 & 0 & 2 \alpha \delta^{\prime}(x-y) & 0 \\
0 & -2 e^{2}(1+\alpha) \delta^{\prime}(x-y) & e^{2}(1+\alpha)^{2} \delta(x-y)-\kappa \delta^{\prime \prime}(x-y) & e \delta^{\prime}(x-y) \\
2 \alpha \delta^{\prime}(x-y) & -e^{2}(1+\alpha)^{2} \delta(x-y)-\kappa \delta^{\prime \prime}(x-y) & 2(\alpha+1) \kappa \delta^{\prime}(x-y) & 0 \\
0 & e \delta^{\prime}(x-y) & 0 & 2 e \delta^{\prime}(x-y)
\end{array}\right),
\end{aligned}
$$

with $\kappa=1+\gamma-\frac{m^{2}}{e^{2}}$. The definition (20), along with equations (21) and (52), enable us to compute the Dirac brackets between the fields describing the reduced Hamiltonian $H_{R}$ :

$$
\begin{gathered}
{\left[A_{1}(x), A_{1}(y)\right]^{*}=\frac{1}{2 e^{2}} \delta^{\prime}(x-y),} \\
{\left[A_{1}(x), \pi_{1}(y)\right]^{*}=\frac{(\alpha-1)}{2 \alpha} \delta(x-y),} \\
{\left[\pi_{1}(x), \pi_{1}(y)\right]^{*}=-\frac{(1+\alpha)^{2}}{4 \alpha e^{2}} \epsilon(x-y) .}
\end{gathered}
$$

From the reduced hamiltonian (51), the following first order equations of motion result in with the use of Dirac brackets (53), (54) and (55).

$$
\begin{gathered}
\dot{A}_{1}=\frac{(\alpha-1)}{2 \alpha} \pi_{1}+\frac{1}{2 \alpha}\left(\gamma-\frac{m^{2}}{e^{2}}+1\right) A_{1}^{\prime}, \\
\dot{\pi}_{1}=\pi_{1}^{\prime}+\frac{e^{2}}{2 \alpha}(\alpha-1)\left(\gamma-\frac{m^{2}}{e^{2}}+2 \alpha+1\right) A_{1} .
\end{gathered}
$$

After a little algebra, the equations (56) and (57) reduce to the following

$$
\begin{gathered}
\partial_{+} A_{1}=\frac{(\alpha-1)}{2 \alpha} \pi_{1}+\frac{1}{2 \alpha}\left(2 \alpha-\gamma+\frac{m^{2}}{e^{2}}-1\right) A_{1}^{\prime} . \\
\partial_{-} \pi_{1}=\frac{e^{2}}{2 \alpha}(\alpha-1)\left(\gamma-\frac{m^{2}}{e^{2}}+2 \alpha+1\right) A_{1} .
\end{gathered}
$$

Here $\partial_{ \pm}$is defined is $\partial_{ \pm}=\partial_{0} \pm \partial_{1}$. The above two equations (58) and (59) ultimately reduce to the following Klein-Gordon Equation

$$
\left(\square-\frac{(\alpha-1)^{2}}{\alpha}\right) \pi_{1}=0,
$$

if we set the same relation (33) in the same manner as it was done in Sec. III. The equation (60), represents a massive boson with square of the mass given by $\tilde{m}^{2}=\frac{-(1-\alpha)^{2}}{\alpha}$. Unlike the previous situation, no massless degrees of freedom appears here. Note that the constraint structure is different and, therefore, disappearance of massless degrees of freedom does not look unnatural. The results reminds us the Mitra and Ghosh's description [18]. We can land on to their results for the specific value of the parameter $\alpha=-1, \gamma=-3$ and $m^{2}=0$. Here, the theoretical spectrum contains only a massive boson with a parameter dependent mass. One can think of it as the photon acquires parameter dependent mass and the fermions of both the chirality have been completely eaten up during the process. 


\section{VERIFICATION OF POINCARÉ ALGEBRA}

We have already mentioned that the gauged lagrangian for chiral boson considered here does not have Lorentz covariance however it is found that the model is embedded with a Lorentz invariant theoretical spectrum. So our next task is to check Poincaré algebra in the reduced phase space. Let us now proceed to check that.

There are three elements in this algebra, the hamiltonian $H_{R}$, the momentum $P_{R}$ and the boost generator $M_{R}$ and they have to satisfy the following relation in $(1+1)$.

$$
\left[P_{r}(x), H_{R}(y)\right]^{*}=0,\left[M_{R}(x), P_{R}(y)\right]^{*}=-H_{R},\left[M_{R}(x), H_{R}(y)\right]^{*}=-P_{R} .
$$

Hamiltonian has already been given in (51) and the momentum density reads

$$
\begin{aligned}
\mathcal{P}_{R} & =\pi_{1} A_{1}^{\prime}+\pi_{\phi} \phi^{\prime}, \\
& =\frac{1}{4 e^{2}} \pi_{1}^{2}+\frac{1}{2}(1-\alpha) \pi_{1} A_{1}^{\prime}+\frac{1}{4} e^{2}(1+\alpha)^{2} A_{1}^{2}
\end{aligned}
$$

The Boost generator written in terms of hamiltonian density and momentum is

$$
M_{R}=t P_{R}+\int d x x \mathcal{H}_{R}
$$

Straightforward calculations shows that equation(61) is satisfied provided the relation (33) between $\alpha, \gamma$ and $m^{2}$ is maintained. The above calculations, therefore, reveals that the physical Lorentz invariance of this model demands the same relation (33) between $\alpha, \gamma$ and $m^{2}$. This certainly strengthens the consistency of the theory under consideration. A closer look reveals that the Lorentz invariance is not maintained in the whole subspace of the theory but in the physical subspace it is maintained in spite of having such a deceptive appearance.

\section{CONCLUSION}

In this paper we have considered the Thirring-Wess model replacing its vector interaction by the chiral one and the model resulted in, is termed here as chiral Thirring-Wess model. Using the standard method of quantization of constrained system by Dirac [28], we have obtained a Lorentz invariant theoretical spectrum provided the relation (33) holds. It is fascinating to mention that this theory contains the ambiguity parameters and the arbitrariness involved in these parameters allows us to set the important relation (33) for these parameters $\alpha$ and $\gamma$ with the classically included parameter $m^{2}$. We have found that the relation (33), became a crucial ingredient to obtain the Lorentz invariant theoretical spectrum. Thus, the physical Lorentz invariance of this theory is achieved here by exploiting suitably the arbitrariness in the ambiguity parameters of regularization. Note that, in [22], a similar approach was made to bring back the lost gauge symmetry with the inclusion of masslike term at the classical level.

If we look at the theoretical spectrum we find that the photon acquires mass like Thirring-Wess model but the mass $\tilde{m}$ is different in this situation. Along with the parameter included at the classical level it also depends on the parameters entered into the theory through the one loop correction. Fermion of a particular chirality gets confined here. It is not surprising since the nature of interaction and the choice of regularization is different in the present situation. We found the similar situation in the Mitra's version of chiral Schwinger model, where he used a regularization that rendered a Gauss's law constraint from which Faddeevian type of Poission bracket resulted in [17, 18].

After imposing a chiral constraint into the proposed action an attempt has been made to obtain a new effective action to describe this new model in terms of chiral boson [32 34]. It is indeed a strange at the same time an interesting aspect of this model that after carrying out investigation on the phase space structure we found a completely different constraint structure from the constraint structure of the model discussed in Sec. III, and consequently, a drastic change in the theoretical spectra resulted in. Photon acquires mass as well and the mass of the photon is also found to be identical to the mass of the massive boson as obtained in the previous case, but the fermions of both the chirality are found to be absent, i.e., confined or eaten up during the process. If we look towards the structure of the theory a deceptive appearance will be observed. To be precise, there is no term in the effective action which had manifestly Lorentz covariant structure. However, physical Lorentz invariance is found to be preserved. The incredible service of the ambiguity parameters through the relation (33) has became a key concerning the maintenance of physical Lorentz symmetry in this case too. So a novel result which follows from this work is that the ambiguity in the regularization renders a remarkable service to make a theory Lorentz invariant getting mixed up suitably with the classical parameter involved in the Proca lagrangian. If it is asked that if the admissible arbitrariness did not permit to set the relation (33) what would be the fate of this model? Simply, it would be disaster. We will not be able to reach into this interesting theoretical spectrum. It will not only loose its exact solvability but loose its physical Lorentz invariance. 
One more point on which we would like to emphasize is that the way the arbitresses of the ambiguity parameter has been exploited here to get back the Lorentz invariance of the model, has not in any, violated any physical principle rather it has helped to maintain the most important physical principle (Lorentz invariance) of a physically sensible theory. Needless to mention, that the technique is more or less standard in $(1+1)$ dimensional QED and Chiral QED. We have witnessed several examples of the use of this mechanism in order to get rescued from different unfavorable as well as un physical situations [11 14, 17, 18, 29 31]. The most remarkable one in this context is the Jackiw and Rajaraman version of chiral Schwinger model [11], where they saved the long suffering of the chiral generation of the Schwinger model [10], from the non-unitary problem.

Acknowledgement: I would like to thank the Director of Saha Institute of Nuclear Physics, Calcutta, and the head of the theory group of the same institute for providing library and computer facilities of the Institute.

[1] J. Schwinger, Phys. Rev. 1282425 (1962)

[2] J. Lowestein and J. A. Swieca, Ann. Phys. 68172 (1971)

[3] S. Coleman, Ann. Phys. 101239 (1976)

[4] A. Casher, J. Kogut and L. Susskind, Phys. Rev. D10 732 (1974).

[5] G. Bhattacharya, A. Ghosh, and P. Mitra, Phys. Rev. D50 4183 (1994)

[6] A. Saha, A. Rahamana and P. Mukherjee, Phys. Lett. B638 292 (2006), Phys. Lett B643 383 (2006)

[7] F. Ardalan, M. Ghasemkhani and N. Sadoohhi, Eur. Phys. J. C71 16062011

[8] W. E. Thirring and J. E. Wess, Ann. Phys. 273311964.

[9] L. V. Belvedere and A. F. Rodrigues, Ann. Phys. 3172005423.

[10] C. R. Hagen, Ann. Phys. (N. Y.) 8167 (1973)

[11] R. Jackiw and R. Rajaraman, Phys. Rev. Lett. 5412191985.

[12] H. O. Girotti, J. H. Rothe and A.K.Rothe Phys. Rev. 33514 (1986)

[13] H. O. Girotti, J. H. Rothe and A.K.Rothe Phys. Rev. 34592 (1986)

[14] K. Harada, Phys. Rev. Lett. 64139 (1990)

[15] S. Miyake and K. Shizuya, Phys. Rev. D46 3781 (1987)

[16] S. Miyake and K. Shizuya, Phys. Rev. D37 2282 (1988)

[17] P. Mitra, Phys. Lett 284231992

[18] S. Ghosh and P. Mitra, Phys. Rev. D44 13321991

[19] S. Mukhopadhyay and P. Mitra, Ann. Phys. (N. Y.) 241681995

[20] S. Mukhopadhyay and P. Mitra, Z. Phys. C67 5251995

[21] P. Mitra and A. Rahaman, Ann. Phys. (N.Y.) 249341996.

[22] A. Rahaman, Mod. Phys. Lett. A29 14500722014

[23] A. Rahaman, Int. Jour. Mod. Phys. A19 30132004

[24] L. D. Faddeev, Phys. Lett. B154 81 (1984)

[25] L. D. Faddeev and S. L. Shatashvili, Phys. Lett. B167 225 (1986)

[26] S. L. Shatashvili, Theor. Math. Phys. 60770 1985, Theor. Mat. Fiz. 60206 (1984)

[27] S. L. Shatashvili, Theor. Math. Phys. 71336 1987, Theor. Mat. Fiz. 7140 (1987)

[28] P. A. M. Dirac, Lectures on Quantum Mechanics(Yeshiva Univ. Press New York, 1964)

[29] R. Banerjee, Phys. Rev. Lett. 561889 (1986)

[30] E. Abdalla, M. C. B. Abdalla, and K. D. Rorhe, 'Two Dimensional Quantum field theory', (World Scientific, Singapore, 1991)

[31] A. Rahaman, Phys. Lett. B697 260 (2011)

[32] W. Siegel, Nucl. Phys. 238307 (1984)

[33] R. Floreanini and R. Jackiw, Phys. Rev. Lett. 6017711988

[34] S. Bellucci, M. F. L. Golterman and D. N. Petcher, Nucl. Phys. B326 3071989

[35] C.Imbimbo and J. Schwimmer, Phys. Lett. 1934451987 